\title{
El RETO DE LA PSICOTERAPIA DE LOS TRASTORNOS DE LA PERSONALIDAD EN DROGODEPENDIENTES
}

\section{The Challenge of Psychotherapy of Personality Disorders in Drug Addicts}

\author{
José Miguel Martínez GonzÁlez ${ }^{1}$ \\ https://doi.org/10.17533/udea.rp.e343631
}

\section{Resumen}

Este artículo de reflexión propone un análisis que permita profundizar en el reto que supone el tratamiento de la drogodependencia en pacientes con trastornos de la personalidad. Se conoce cada vez mejor la especificidad de variables que influyen significativamente en el tratamiento de estos pacientes. Variables como el craving, estrategias de afrontamiento, autoeficacia, creencias nucleares relacionadas con el consumo, calidad de vida, autoengaño o motivación, tienen gran repercusión en su evolución y resultado. La especificidad del tratamiento en estos casos exige prestar especial atención al modo en que se comportan estas variables en comparación con lo que sucede

\section{Abstract}

This reflection article proposes an analysis that allows us to delve into the challenge posed by drug addiction treatment in patients with personality disorders.

The specificity of variables that significantly influence the treatment of these patients is

Recibido: 2020-06-10 / Aceptado: 2020-12-14

Para citar este artículo en APA: Martínez González, J. M. (2020). El reto de la psicoterapia de los trastornos de personalidad en drogodependientes. Revista de Psicología Universidad de Antioquia, 12(2), e343631. doi: https://doi.org/10.17533/ udea.rp.e343631 cuando el paciente no presenta esta psicopatología comórbida.

Podemos afirmar, con los datos de los que disponemos en la actualidad, que los drogodependientes con trastorno de la personalidad pueden beneficiarse del tratamiento tanto como lo hacen aquellos sin comorbilidad, cuando se llevan a cabo intervenciones sustentadas en componentes esenciales bien definidos, adaptados a esta comorbilidad psicopatológica y durante el tiempo necesario.

Palabras clave: tratamiento, trastornos de la personalidad, drogodependencia, efectividad, cognitivo-conductual.

becoming better known. Variables such as craving, coping strategies, self-efficacy, core beliefs related to consumption, quality of life, self-deception or motivation, have a great impact on its evolution and outcome. The specificity of the treatment in these cases

1 Especialista en psicología clínica. Centro Provincial de Drogodependencias de Granada, España. Correo: jmmgonz@dipgra.es; https:// orcid.org/0000-0002-7960-2922. 
requires paying special attention to the way in which these variables behave in comparison with what happens when the patient does not present this comorbid psychopathology. We can affirm with the data that we currently have, that drug addicts with personality disorder can benefit from treatment as much as those without comorbidity, when interventions based on well-defined essential components are carried out, adapted to psychopathological comorbidity and for the necessary time.

Keywords: treatment, personality disorders, drug dependence, effectiveness, cognitivebehavioral.

\section{Abordaje de los trastornos de la personalidad}

La reflexión que estructura este artículo responde a las inquietudes clínicas e investigativas que han marcado nuestra línea de investigación desde su comienzo hace años. Las dificultades que entraña el tratamiento de las adicciones cuando los pacientes presentan de forma comórbida un trastorno de la personalidad, pueden vencerse implantando psicoterapias que cuenten con estrategias capaces de generar los cambios necesarios para que la persona esté protegida ante posibles recaídas en el consumo de drogas y de mejorar sensiblemente en lo referente a su TP. Las propuestas terapéuticas que se exponen se sustentan en los resultados de investigaciones que a lo largo del tiempo han abordado diferentes aspectos claves de la psicoterapia y variables determinantes en el resultado del tratamiento de estos pacientes. Nuestra trayectoria nos permite poner en valor la utilidad de los resultados de los estudios realizados (Becoña, López y Martínez-González, 2020; Becoña et al., 2011; Martínez-González, 2011; Martínez-González, Albein-Urios, Lozano-Rojas y Verdejo-García, 2014; 2015; Martínez-González, Caracuel, Vilar, Becoña y Verdejo-García, 2020; Martínez-González, Graña y Trujillo, 2009; 2011; Martínez-González y Verdejo-García, 2011; 2012; 2014; Martínez-González ,Verdejo-García y Becońa, 2012; Martínez-González, Vilar-López, Becoña y Verdejo-García, 2016; 2018), en la medida en que pueden mejorar significativamente los programas de tratamiento que se lleven a cabo con estos pacientes.

A lo largo de los últimos años hemos sido testigos de la evolución que ha experimentado el tratamiento de los trastornos de la personalidad (TP). Hemos transitado de un espacio en el que la sospecha de psicopatología de la personalidad invalidaba cualquier intento de actuación terapéutica por considerarla 
totalmente ineficaz (Rousanville, Dolinsky, Sabor y Meyer, 1987), a evaluar a cada paciente con la especificidad que permite identificar esta patología con suficiencia, para así intervenir con estrategias efectivas. Ya no se discute el hecho de que el tratamiento de drogodependientes con TP se diferencia significativamente del que se lleva a cabo cuando no presentan esta comorbilidad (Martínez-González, 2011).

Las investigaciones en torno a la vinculación de la presencia de TP con determinados patrones de consumo o sustancias específicas insisten en que no es posible establecer una relación de exclusividad entre ambas condiciones al hallar su vínculo. Esto es, diferentes TP se observan entre pacientes que consumen distintos tipos de sustancia y en los cuales se presentan patrones de consumo que pueden variar de acuerdo con la historia adictiva de cada persona.

La utilización de instrumentos adecuados para el diagnóstico de los TP en drogodependientes, la experiencia de los profesionales y la normalización del tratamiento de esta población en los centros donde se lleva a cabo, ha permitido mejorar los estudios de incidencia de esta clase de trastornos entre pacientes que solicitan ayuda. La incidencia de тP en drogodependientes es bastante más elevada de lo que se observa en población normal. Respecto a la última, mientras que en algunas sociedades se ha identificado hasta en un $15 \%$, en drogodependientes la incidencia alcanza alrededor del 50\% (Bravo, Echeburúa y Azpiri, 2008; Martínez-González, 2011).

La epidemiología descrita en diferentes fuentes depende del grupo poblacional en el que se realiza el estudio; si la población se encuentra en recurso asistencial, ambulatorio, o en un régimen de internamiento o reclusión, donde se ha llegado a identificar hasta un 70\% de internos con тP (Marset y Ritter, 2004). Si nos referimos a estudios realizados en centros de tratamiento ambulatorio, probablemente podríamos observar un perfil psicopatológico diferente al que encontramos en aquellos realizados en centros de internamiento, entre los que cabe esperar, como se señaló, mayor incidencia de esta psicopatología. La variabilidad epidemiológica que hallamos en cada uno de los tipos de centros de tratamiento debe comprometernos con una evaluación minuciosa de cada paciente sin asumir la presencia de un tipo de tp por el solo hecho de que este consuma una determinada sustancia. 


\section{Evaluación}

La utilización de un instrumento de evaluación adecuado determina parte del éxito de esta, especialmente si tenemos en cuenta que, en este caso, la valoración de la personalidad en drogodependientes podría verse dificultada por el solapamiento de determinados síntomas, que pueden confundir al profesional respecto del verdadero origen de ellos. Esto es, la evaluación debe sortear la influencia que el consumo de drogas pueda ejercer en la valoración de la personalidad y así evitar que los síntomas propios del тP puedan interpretarse como parte de la expresión de la drogadicción o viceversa. En este sentido, el instrumento indicado en estos casos es la entrevista clínica. Se sugieren: la International Review of Personality Disorders (López-Ibor y Pérez y Rubio, 1996) o la SCID-II (First, Spitzer, Gibbson y Williams, 1998).

No identificar la presencia de este trastorno trae consigo problemas de calado que impiden llevar a cabo una intervención con garantías. Cuando coexiste con un TP, el hecho de intervenir solamente en la adicción acarrea, entre otros problemas: el abandono del tratamiento; la imposibilidad para explicar suficientemente las razones por las que un paciente en abstinencia vuelve a consumir; el deterioro progresivo de la relación terapéutica; la aparición de estrés asistencial; el hecho de que el paciente no pueda seguir las indicaciones terapéuticas; las elecciones erróneas de estrategias terapéuticas o recursos asistenciales; desestimar el uso de psicofármacos, o hacerlo erróneamente; y, cómo no, la incompetencia de los familiares, traducida en confrontación y falta de apoyo en el tratamiento, al no interpretar las conductas disruptivas en el contexto de la psicopatología (Martínez-González, 2011).

Es evidente la extraordinaria importancia que tiene identificar la comorbilidad con esta psicopatología lo antes posible, con el objetivo de orientar las intervenciones de acuerdo con la personalidad y las particularidades clínicas de cada caso. La presencia de TP introduce innumerables matices en la relación terapéutica y, en definitiva, en los mecanismos que potencian la adherencia al tratamiento, especialmente en las primeras semanas, que es cuando esta se construye y lleva a predecir el resultado del tratamiento (Martínez-González y Verdejo-García, 2014). 


\section{Marco teórico}

La capacidad de llevar a cabo tratamientos efectivos depende en gran medida del encuadre asistencial en el que se atiende al paciente. Para aquellas zonas geográficas en las que la asistencia de las adicciones y la que se presta a la salud mental se realiza por instituciones diferentes, debe cuidarse especialmente la coordinación entre los profesionales de ambas redes asistenciales, pero, sobre todo, hacer lo posible por implementar tratamientos integrados. El modelo de atención integral a los pacientes con patología dual implica que sea un solo servicio asistencial quien se ocupe del tratamiento del paciente, evitando escindir un fenómeno clínico indivisible (Becoña et al., 2020). Así, la intervención que se desarrolle en un centro especializado para el tratamiento de las adicciones debe ocuparse simultáneamente de la psicopatología comórbida.

Es necesario que los profesionales cuestionemos nuestro modelo de intervención, y los recursos de tratamiento en general, para revisar los principios heredados que pueden influir negativamente, por la incomprensión de una psicopatología comórbida, en la implicación del terapeuta. Parece que es momento de desterrar determinadas aseveraciones de un marco teórico imperante que a lo largo de los años mostró una realidad clínica parcial, y seguramente carente de estrategias, ahora puestas en cuestión con el esfuerzo de los profesionales y las investigaciones. Algunas de estas premisas son las siguientes:

- Si históricamente se asumió que estos pacientes presentaban mayor número de consumos a lo largo del tratamiento (First y Gladis, 1996) por su patología dual, lo que impedía avanzar en la estabilidad de la abstinencia y en la mejoría de los rasgos de la personalidad, se ha visto en algunos trabajos que a lo largo del tratamiento no existen diferencias significativas en este aspecto entre los pacientes, en función de la presencia de тP (Martínez-González et al., 2011).

- Si bien se consideraron intratables los тP (Vaillant y Perry, 1980), existen estudios que muestran que su diagnóstico no es predictor del resultado del tratamiento de la drogadicción, por lo que cabe pensar que la actuación terapéutica neutraliza el impacto que esta psicopatología pueda ejercer sobre este tratamiento (Martínez-González et al., 2009). 
- Se ha asumido durante mucho tiempo que los pacientes con TP eran pacientes poco motivados para el tratamiento; ahora bien, se ha demostrado, por el contrario, que pueden incluso presentar niveles de motivación más elevados a los informados por drogodependientes sin esta psicopatología (van Beek y Verheul, 2008).

- Si bien se asumía que el tratamiento de los tp fracasaba (Vaillant y Perry, 1980), se ha podido constatar cómo la psicoterapia cognitivo-conductual es efectiva en el tratamiento de estos pacientes (Martínez-González et al., 2018).

Estas cuestiones son las que debemos revisar en nuestra práctica clínica, con la intención de potenciar alternativas terapéuticas que están permitiendo llevar a cabo tratamientos efectivos en drogodependientes con TP.

Para la fortaleza de un modelo actual de tratamiento, y como consecuencia, de su objetivo terapéutico, este debe contemplar comprensivamente las enormes diferencias entre los diferentes TP e incorporar estrategias en consonancia con un programa de intervención promotor de expectativas positivas sobre el pronóstico. De otro modo, no se puede transmitir a un paciente la confianza que necesita para encarar un trayecto terapéutico difícil, largo, costoso, confuso, y, en ocasiones, oscilante entre el progreso y el retroceso. Solo la confianza del paciente en el terapeuta, construida en la exposición de un modelo de intervención coherente, estructurado y persistente, a pesar de las dificultades, puede ayudar a los pacientes drogodependientes con TP. La esperanza en la posibilidad de ayudar a personas con una terapéutica cada vez más estudiada puede contribuir a explicar el hecho de que investigaciones actuales informen resultados satisfactorios, en comparación a lo que sucedía hace años. Hoy en día contamos con trabajos que describen suficientemente las líneas terapéuticas más útiles en este campo y por tanto deberían orientar las intervenciones que se lleven a cabo en nuestros centros de tratamiento (Becońa et al., 2020; Martínez-González y Verdejo-García, 2014). 


\section{La efectividad de la psicoterapia}

Desde hace años disponemos de investigaciones que han contribuido a mejorar la comprensión del cuadro clínico cuando un drogodependiente presenta simultáneamente un Tr. Tal es así que las expectativas de los profesionales respecto a la utilidad de lo que hacen, y esperamos que por extensión las de los propios pacientes, se dirigen a un horizonte esperanzador, al asumir que estos pueden mejorar significativamente con psicoterapia.

Diversos estudios muestran que la psicoterapia es eficaz para el tratamiento de estos pacientes (Perry y Bond, 2002), habiéndose constatado el mantenimiento de la mejoría en las relaciones interpersonales, hospitalizaciones y funcionamiento general (Simon y Lambert, 2010). En relación con los componentes que sustentan esta efectividad se han identificado cuatro: la psicoeducación, la intervención motivacional, la intervención cognitiva y las relaciones interpersonales (Hadjipavlou y Ogroduiczuk, 2010). Las intervenciones que logran mejoras significativas en los TP se ocupan de la conciencia y conocimiento que el paciente tenga de su trastorno, de su motivación para realizar el tratamiento y su disposición al cambio, y de la modificación de esquemas de creencias que afectan el modo en que se ve así mismo y su entorno, así como de la relación que establece con otras personas. De este modo, las terapias psicológicas que han demostrado ser efectivas en los TP son aquellas que: están bien estructuradas, enfatizan la implicación de paciente y terapeuta, tienen objetivos claros, son coherentes para paciente y terapeuta, son relativamente largas en el tiempo, persiguen una fuerte cooperación entre terapeuta y paciente, y se integran en otros servicios para este último (Bateman y Fonagy, 2000). Así, puede decirse que se conocen los componentes que deberían dirigir nuestras intervenciones, con la debida temporización que exige individualizar cada uno de los tratamientos, porque cada paciente necesita diferente tiempo para conseguir los objetivos terapéuticos. Todo esto dependerá, sin duda, de la incidencia que pueda ejercer en esa evolución el consumo de sustancias a lo largo del tratamiento.

En el caso de pacientes drogodependientes con TP, los componentes esenciales del programa de intervención giran en torno a: la evaluación del riesgo 
a lo largo de todo el proceso; el desarrollo del tratamiento como un paquete integrado en el que se le presta especial atención, al inicio del mismo, a la motivación y a los procesos de validación; otorgar gran peso a la adherencia para evitar los abandonos; centrar la atención en los rasgos de la personalidad maladaptativos, especialmente, en las habilidades cognitivas y de afrontamiento, porque de ese modo mejora la sintomatología y disminuye el riesgo de recaída; proporcionar suficiente estructura y seguridad a los pacientes; priorizar la intervención motivacional y de los problemas interpersonales a lo largo de todo el tratamiento, a la vez que se incorporan cuidados posteriores a este (van den Bosch y Verheul, 2007; Verheul, van den Bosch y Ball, 2007). Varias investigaciones nos han permitido comprobar la utilidad de estos componentes (Martínez-González et al., 2009; Martínez-González et al., 2011; Martínez-González et al., 2018).

Sin duda, la efectividad depende de la adherencia terapéutica, puesto que de esa manera los pacientes permanecen en tratamiento el tiempo suficiente (Becoña et al., 2011). Al respecto, un estudio nos permitió comprobar que esta psicopatología no puede vincularse sin más con un mayor riesgo de abandono al iniciar el tratamiento (Martínez-González et al., 2014). Así, las actuaciones que potencian la adherencia son tan importantes en estos pacientes como en el resto de los drogodependientes, porque a la luz de las investigaciones, cuando se cuidan los aspectos que la mejoran, se obtienen resultados satisfactorios en el tratamiento.

Las aportaciones de numerosas investigaciones con resultados prometedores, junto a nuestra propia experiencia clínica, nos permitió elaborar un material con un enfoque eminentemente práctico para el tratamiento de drogodependientes con тр (Martínez-González y Verdejo-García, 2014). En él se detallan, además de las claves que facilitan una adherencia terapéutica satisfactoria, diferentes propuestas para que el terapeuta afronte los retos que presentan estos casos, los componentes que son esenciales en los programas de prevención de recaídas, un conjunto de técnicas específicas y particularmente indicadas para cada uno de los TP, orientaciones terapéuticas que incorporan colaborativamente a la familia, y técnicas psicológicas que permiten modular las alteraciones neuropsicológicas descritas en estos pacientes. 
A continuación, se detallan algunas de nuestras investigaciones sustentadas en un modelo cognitivo-conductual de prevención de recaídas, que nos han permitido conocer mejor las estrategias más adecuadas para intervenir sobre variables relevantes en el tratamiento de drogodependientes con тP.

\section{Variables de interés clínico}

Las variables que se mencionarán ocupan un papel central en el tratamiento de las adicciones, debiéndoles prestar suficiente atención en el desarrollo de los programas de prevención de recaídas cuando el drogodependiente presenta también un TP. Todo indica que la especificidad de la intervención es posible en la medida en que el tratamiento se ocupa diferencialmente de estas variables, según se trate o no de un paciente con esta patología dual, y por supuesto contemple las diferencias que introduce cada uno de los TP.

Craving. Sin duda es la variable que probablemente más investigaciones ha generado en el ámbito de las adicciones, y de forma especial en los procesos de recaída (Martínez-González et al., 2012). Por este motivo, la comprensión y gestión del deseo de consumo ocupa una parte importante de los programas de prevención de recaídas. Investigaciones previas (Martínez-González et al., 2011) nos permitieron describir las fluctuaciones de esta variable a lo largo del proceso de recuperación en función de la presencia de TP. Si bien se sabe que la intensidad del deseo de consumo desciende a lo largo del tiempo en que el paciente permanece abstinente, los que presentan un TP informan niveles de craving más estables a los tres meses de iniciado el tratamiento. Comparativamente, se observa que los pacientes que no presentan esta psicopatología experimentan disminuciones significativas del deseo de consumo en ese mismo periodo. Este patrón observable entre pacientes con TP, que puede depender también de otros aspectos como las creencias nucleares relacionadas con el consumo de drogas, debe tenerse en cuenta sobre todo durante los primeros meses de tratamiento, donde puede fluctuar significativamente el deseo de consumo.

Estrategias de afrontamiento. Los pacientes con TP no solo presentan de forma significativa menor nivel de autocontrol, habilidades sociales, optimismo y 
autoestima, sino que a su vez se destacan en la utilización de estrategias orientadas a resolver de forma inmediata el malestar emocional cuando es necesaria la resolución de un problema (Martínez-González et al., 2015). Esto implica que tienden a utilizar más estrategias dirigidas al afrontamiento emocional, reforzando modelos de afrontamiento potenciados por la propia adicción, es decir, en la medida en que el drogodependiente continúa recurriendo a estrategias de este tipo, fortalece mecanismos de afrontamiento centrados en el uso de sustancias para la evitación del malestar emocional. En consecuencia, el paciente desestima el uso de estrategias centradas en la tarea para la solución de problemas, que es la alternativa deseable.

Cabe mencionar el peso que tiene el sistema atribucional en el proceso de recuperación, porque en la medida en que el locus de control es más externo, disminuye la implicación necesaria del paciente en las propuestas terapéuticas. Los pacientes con TP se diferencian también del resto de drogodependientes en esta variable (Martínez-González et al., 2015). Por esta razón, el entrenamiento en estrategias de resolución de problemas debe convertirse en diana terapéutica en estos casos, porque con su mejoría pueden abordarse con éxito otras conductas esenciales en la adaptación del individuo a su entorno.

Autoeficacia. Es conocida la relevancia de las expectativas de autoeficacia en el proceso de recuperación de una drogadicción. Estudios en población de drogodependientes con TP indican que al inicio del tratamiento estos pacientes presentan niveles de autoeficacia menores en comparación al resto (Llorente e Iraurgi, 2008). Esto tiene una enorme repercusión en la intervención porque, entre otros aspectos, este tipo de expectativas tienen gran influencia en el impacto de determinados síntomas en el paciente, particularmente en cuanto a su intensidad, lo cual puede ser clave para afrontar aquellos vinculados con la adicción y el TP (Martínez-González et al., 2015; Martínez-González y Verdejo-García, 2012). Por este motivo, los programas de intervención deberían ocuparse de mejorar lo antes posible las expectativas de autoeficacia, dado que estos pacientes no solo deben afrontar los síntomas propios del proceso de cambio en las adicciones, sus dificultades y riesgos de recaída, sino también los síntomas propios de cada TP. Su afrontamiento influye sin duda en la abstinencia del paciente (Martínez-González y Verdejo-García, 2012). 
Creencias nucleares. Varias investigaciones permitieron verificar que el grado con el que los pacientes se identifican con creencias nucleares relacionadas con la adicción, como por ejemplo: "la vida sin drogas es aburrida", o con la propia experiencia de deseo, como por ejemplo: "me es difícil hacer frente al deseo de consumir”, predice el nivel de craving (Martínez-González y Verdejo-García, 2011; Martínez-González et al., 2012). A su vez, en la medida en que los pacientes se identifican más con estas creencias, se potencia un locus de control externo que, como se planteó previamente, puede afectar de manera negativa al resultado del tratamiento (Martínez-González et al., 2015). El análisis de la evolución de estas creencias a lo largo del tratamiento mostró que el nivel de identificación de los pacientes con Tp era más irregular que el observado en el grupo de pacientes sin esta psicopatología, el cual parecía seguir una evolución esperable de acuerdo con el contenido de las creencias (Martínez-González y Verdejo-García, 2012). En definitiva, se pudo constatar que los pacientes con TP presentaban más dificultad para modificar determinadas creencias nucleares, posiblemente responsables en gran medida de la aparición de craving. Este resultado implica prestar especial atención, en el transcurso de la intervención, al grado de identificación con estas creencias de los pacientes con TP, para de este modo no asumir como general la estabilidad de determinados logros que pueden observarse en drogodependientes sin esta comorbilidad.

Calidad de vida. La calidad de vida se ha utilizado como indicador del resultado del tratamiento de las drogodependencias. Al iniciarlo, los pacientes con TP informan niveles menores en comparación a los que no presentan esta psicopatología (Martínez-González et al., 2011), por ello debe evaluarse qué consigue el tratamiento en relación con esta variable. La investigación mencionada mostró que a pesar de que el nivel de calidad de vida de los pacientes con тP al iniciar el tratamiento es menor, mejoró significativamente respecto a la evaluación inicial, a los tres y doce meses. De esta manera, pudo verse que estos pacientes logran beneficiarse del tratamiento tal como lo hacen aquellos sin la patología dual, mejorando significativamente la percepción de calidad de vida.

Motivación. Esta puede caracterizarse mediante dos dimensiones: la motivación para el tratamiento y la motivación para el cambio. Tradicionalmente se ha asociado a los pacientes con TP con una baja motivación, o lo que es 
peor, esta se ha interpretado como un rasgo inherente a la personalidad (Reich y Vasile, 1993). Sin embargo, lo anterior no es coherente con estudios que no solo niegan la existencia de esta diferencia en el nivel motivacional, sino que incluso refieren que estos pacientes pueden presentar niveles significativamente mayores (Martínez-González et al., 2020; van Beek y Verheul, 2008). Estos resultados ponen de relieve la importancia de interpretar la motivación como una variable dinámica a lo largo del tratamiento, que no empeora necesariamente por el solo hecho de que el drogodependiente presente un тр.

Autoengaño. Si bien se sabe que los pacientes drogodependientes presentan niveles de autoengaño más elevados a los observados en la población general, esta diferencia se acentúa aún más cuando presentan un TP (Martínez-González et al., 2016). En la investigación citada pudimos comprobar que las creencias nucleares relacionadas con el consumo y el craving, que se vinculan de forma significativa con la aparición del deseo de consumo, también predicen el nivel de autoengańo, siendo mayor en la medida en que las personas se identifiquen más con ese tipo de creencias. Por otro lado, sumado al impacto del autoengaño en diferentes áreas de la vida de las personas, en drogodependientes se ha asociado a periodos más breves de abstinencia.

El resultado de todos estos trabajos nos ha llevado a fortalecer la convicción de que los drogodependientes con TP pueden beneficiarse del tratamiento para superar su adicción, tanto como lo hacen los demás. Estos logros dependen claramente de la adaptación de los componentes esenciales que se han asociado con el éxito de cada tratamiento y la atención singularizada que requieren los TP, por ejemplo, en la intervención sobre las variables de interés clínico descritas.

\section{Retos futuros para un tratamiento eficaz}

Existen varios retos importantes que debemos asumir. Uno de ellos es la adaptación de los recursos para el tratamiento de las drogodependencias a las necesidades terapéuticas que exigen los pacientes con TP. Parece fuera de toda duda que estos pacientes deben seguir tratamientos adaptados, por ejemplo en recursos de internamiento, sin que tal adaptación afecte a la convivencia 
general entre los usuarios. En el caso de las intervenciones que se llevan a cabo en régimen ambulatorio, es bastante fácil efectuar modificaciones. La cuestión de fondo es disponer de la flexibilidad requerida para ajustar las intervenciones terapéuticas en el tiempo necesario y con la especificidad que necesita cada paciente. La viabilidad de las adaptaciones depende no solamente de la organización del recurso en sí mismo, sino también de la preparación que cada profesional tenga en el ámbito de la psicología clínica. No cabe espacio para la improvisación cuando ya se conocen los aspectos que más afectan a los drogodependientes con TP. Pongamos por ejemplo la latencia y duración de las sesiones, la implicación de la familia y, sobre todo, la utilización de una terapéutica que sea específica para cada тр.

Debemos evitar la estandarización indiscriminada de modalidades terapéuticas que solo consiguen asistir a los pacientes que de antemano pueden rentabilizarlas, entre otras razones, por no presentar un TP. No es el paciente el que debe adaptarse a nosotros y no es razonable que justifiquemos de forma generalizada los abandonos de tratamiento con la falta de motivación de estos. Deberíamos sopesar la parte de responsabilidad que nos atañe, tanto desde el punto de vista de la adaptación terapéutica del recurso - tan necesario en los casos con patología dual- como por la modalidad terapéutica utilizada.

Además, la adaptabilidad de recursos pasa necesariamente por la implicación coordinada de un equipo multidisciplinar de profesionales. Si las áreas psicológica, médica y social desarrollan sus intervenciones de forma autónoma, corremos el riesgo de potenciar determinadas actuaciones no necesarias o incluso contraindicadas, en detrimento de otras imprescindibles. Cada intervención debiera estar a cargo de los profesionales que cuentan con los niveles de competencias necesarios para la atención de la psicopatología. La contribución inestimable de aquellos profesionales no especializados no puede variar la propuesta terapéutica que se diseñe desde la especialización en el tratamiento de la adicción y de psicopatologías comórbidas.

Otro reto es el uso racional de los psicofármacos. El soporte farmacológico puede favorecer la evolución del tratamiento si se usa oportunamente, porque la perdurabilidad de algunos síntomas, habitual en este tipo de pacientes, puede fácilmente invitar a usar durante largo tiempo fármacos para manejar estos 
síntomas que generalmente son parte de rasgos de la personalidad o se derivan de ellos. Esto sucede con la sintomatología depresiva, ansiosa o la impulsividad. En síntesis, debemos cuidar especialmente el uso de fármacos cuando los síntomas son parte de la psicopatología y por tanto su evolución depende del proceso que sigue el trastorno en sí. La psicoterapia puede complementarse con la utilización de fármacos para mejorar la respuesta inicial a las estrategias terapéuticas que se quieren implantar, pero el empleo de medicación no debería eclipsar las estrategias psicológicas que la persona debe interiorizar. El uso de fármacos poco supervisado, o bajo parámetros apropiados para personas que no tienen problemas con las drogas, puede desencadenar adicciones yatrogénicas con más facilidad. Por estas razones, es responsabilidad de los equipos de profesionales, que tratan a un drogodependiente con TP, sustentar solo parcialmente su intervención en el efecto de los fármacos, durante el tiempo imprescindible y de forma coordinada con la intervención psicológica (Marín y Fernández, 2007; Martínez-González y Verdejo-García, 2014; Peláez, Reyes-Molón y Teijeira-Levet, 2013).

Un reto que enfrentamos los profesionales que trabajamos en el ámbito de las adicciones es llevar a cabo investigaciones con muestras suficientemente amplias, que permitan discriminar aspectos relevantes de la psicoterapia, cuando un drogodependiente presenta a su vez un TP. Los estudios que se realizan en centros de tratamiento frecuentemente cuentan con muestras de pacientes reducidas, sujetas al tipo de población atendida en cada centro en el que se realiza el estudio. Como consecuencia de ello, los análisis solo se centran en determinados perfiles de pacientes. Una manera de resolver este problema es desarrollar diseños multicéntricos para estudiar grupos de pacientes con el número de integrantes necesario y un mismo perfil psicopatológico. Históricamente, el apoyo a los profesionales por parte de las instituciones dedicadas al tratamiento de las adicciones para el desarrollo de investigaciones ha sido deficiente, conllevando un retraso terapéutico innecesario, sustancialmente perjudicial para los usuarios de los centros de tratamiento.

Pronto nos encontraremos con un reto que no será menor, esto es, la incorporación de la nueva propuesta de la CIE-11 respecto a los TP (Organización Mundial de la Salud [oms], 2019). La nueva conceptualización abandona la 
clasificación categorial para desarrollar un modelo dimensional sustentado en rasgos o patrones de personalidad prominentes, como: afectividad negativa, desapego, comportamiento disocial, desinhibición, características anancásticas y patrón límite. Estos patrones pueden estar presentes en los niveles grave, moderado y leve. La cuestión es el modo en que aplicaremos esta nueva forma de entender la psicopatología de la personalidad, porque utilizar dimensiones para describirla puede sin duda mejorar nuestra comprensión en algunos aspectos, pero también puede retrasar enormemente las actuaciones terapéuticas, que es lo que sucede cuando no se identifica un TP, como trastorno que condiciona la vida del paciente. Parcelar el análisis del todo que representa el cuadro clínico, puede llevarnos a intervenir sobre síntomas (rasgos) de forma aislada, como si la evolución de cada uno de ellos no dependiera del resto. El desafío, por tanto, es no caer en este tipo de errores, para evitar, entre otras cosas y como ya se ha mencionado anteriormente, encomendar a determinados fármacos corregir lo que solo puede modificarse con estrategias psicológicas.

\section{Conclusión}

Todo parece indicar que el éxito del tratamiento depende en gran medida de nuestras expectativas, porque a los pacientes les transmitimos la esperanza de superar su trastorno si nosotros creemos que es posible (Martínez-González y Verdejo, 2014). El tratamiento guiado por la personalidad, y sustentado en estrategias terapéuticas indicadas según cada caso, ha mostrado la capacidad de mejorar significativamente la vida de los pacientes. Recientemente se ha publicado una monografía sobre esta cuestión, en la que los autores manifestaron que la evidencia de varios ensayos controlados y aleatorios, con los cuales se demostró la efectividad de diversas psicoterapias (p. ej.: la terapia dialéctica conductual [DBT] y el tratamiento basado en la mentalización [мвт]), junto con los hallazgos de varios estudios longitudinales, sugiere que tal aumento del optimismo clínico está justificado (Kealy y Ogrodniczuk, 2018).

No deberíamos dejarnos llevar por el desánimo derivado de las dificultades que trae consigo el tratamiento de estos pacientes, evitando asociar dificultad con imposibilidad. Debemos ayudar no solo a los pacientes que quieren dejar 
de consumir y cuentan con los recursos personales para lograrlo, sino también a aquellos que quieren superar su adicción, pero por su psicopatología comórbida tienen grandes dificultades para conseguirlo.

\section{Referencias}

Bateman, A. y Fonagy, P. (2000). Effectiveness of Psychotherapeutic Treatment of Personality Disorder. British Journal of Pschiatry, 177, 138-143. http://dx.doi. org/10.1192/bjp.177.2.138

Becoña, E., Cortés, M., Arias, F., Barreiro, C., Berdullas, J., Iraurgui, I., Llorente, J. M., López, A., Madoz, A., Martínez-González, J. M., Ochoa, E., Palau, C., Palomares, A., y Villanueva, V. (2011). Manual de adicciones para psicólogos especialistas en psicología clínica en formación. España: Socidrogalcohol.

Becońa, E. López, A. y Martínez-González, J. M. (2020). Cocaína, cannabis y heroína. España: Síntesis.

Beek, N. van y Verheul, R. (2008). Motivation for Treatment in Patients with personality Disorders. J. Pers. Disord., 22, 89-100. doi: https://doi.org/10.1521/ pedi.2008.22.1.89.

Bosch, L. van den y Verheul, R. (2007). Patients With Addiction and Personality Disorder: Treatment Outcomes and Clinical Implications. Current Opinion in Psychiatry, 20,67-71. doi:http://dx.doi.org/10.1097/YCO.0b013e328011740c

Bravo, R., Echeburúa, E. y Azpiri, J. (2008). Diferencias de sexo en la dependencia del alcohol: dimensiones de personalidad, características psicopatológicas y trastornos de personalidad. Psicothema, 2, 218-223.

First, M. B. y Gladis, M. M. (1996). Diagnóstico y diagnóstico diferencial de los trastornos psiquiátricos y por uso de sustancias. En J. Solomon, S. Zimberg y E. Shollar (eds.), Diagnóstico Dual (pp. 41-56). España: Ediciones en Neurociencias.

First, M. B., Spitzer, R. L., Gibbson, M. y Williams, J. B. W. (1998). SCID-II. Guia del usuario de la entrevista clínica estructurada para los trastornos de personalidad del eje II del DSM-IV. Masson.

Hadjipavlou, G. y Ogroduiczuk, K. (2010). Promising Psychotherapies for Personality Disorders. Canadian Journal of Psychiatry, 55, 202-210.

Kealy, D. y Ogrodniczuk, J. S. (2018). Mechanisms of Change in Treatments of Personality Disorders: Commentary on the Special Section. Journal of 
Personality Disorder, 32(Suppl.), 134-142. doi: https://doi.org/10.1521/ pedi.2018.32.supp.134.

Llorente, J. J. e Uraurgi, E. I. (2008). Tratamiento cognitivo conductual aplicado en la deshabituación de cocaína. Trastornos Adictivos, 10, 252-274.

López-Ibor, J., Pérez, A., y Rubio, V. (1996). Examen Internacional de los Trastornos de la Personalidad; módulo DSM-IV [International Review of Personality Disorders; DSM-IV Module] (versión española). Madrid: Organización Mundial de la Salud.

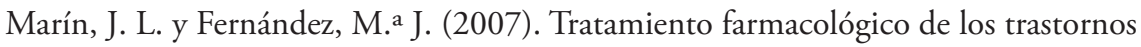
de la personalidad. Clínica y Salud, 18, 259-285.

Martínez-González, J. M. (2011). Drogodependencias y trastornos de la personalidad: variables relevantes para su tratamiento. Papeles del Psicólogo, 32, 166-174.

Martínez-González, J. M., Albein-Urios, N., Lozano-Rojas, O., y Verdejo-García, A. (2014). Aspectos diferenciales del riesgo de abandono al inicio del tratamiento de la adicción a la cocaína en pacientes con trastornos de la personalidad. Adicciones, 26(2), 116-125. doi: http://dx.doi.org/10.20882/adicciones.13

Martínez-González, J.M., Albein-Urios, A., Lozano-Rojas, O., y Verdejo-García, A. (2015). Variables de interés clínico en el tratamiento cognitivo-conductual de la adicción a la cocaína: la especificidad de los trastornos de la personalidad. Revista de Psicopatología y Psicología Clínica, 20, 115-123. doi: http://dx.doi. org/10.5944/rppc.vol.20.num.2.2015.15166

Martínez-González, J. M., Caracuel, A., Vilar, R., Becoña, E., y Verdejo-García, A. (2020). Evaluation of Motivation for the Treatment of Drug Dependent Patients with Personality Disorders. Spanish Journal of Psychology, 23, E15. 1-9. doi: https://doi.org/10.1017/SJP.2020.13

Martínez-González, J. M., Graña, J. L., y Trujillo, H. (2009). Influencia de los trastornos de la personalidad y patrones de consumo en la eficacia de un programa de prevención de recaídas para el tratamiento del alcoholismo. Adicciones, 21, 105-112. doi: http://dx.doi.org/10.20882/adicciones.237

Martínez-González J. M., Graña J. L., y Trujillo, H. (2011). Estudio longitudinal sobre calidad de vida, craving y ajuste psicológico en pacientes dependientes del alcohol: variaciones en función de los trastornos de la personalidad. Adicciones, 23, 227-235.

Martínez-González, J. M. y Verdejo-García, A. (2011). Creencias básicas adictivas y craving. Adicciones, 23, 339-352. 
Martínez-González, J. M. y Verdejo-García, A. (2012). Evolución de las creencias nucleares relacionadas con la adicción en drogodependientes con y sin trastornos de personalidad. Adicciones, 24, 229-238. doi: http://dx.doi.org/10.20882/ adicciones.94

Martínez-González, J. M. Verdejo-García, A., y Becoña, E. (2012). Estudio exploratorio del impacto de las creencias nucleares relacionadas con la adicción en el consumo tras la abstinencia. Trastornos Adictivos, 14, 105-111.

Martínez-González, J. M. y Verdejo-García, A. (2014). Drogodependientes con trastornos de la personalidad. Guía de intervenciones psicológicas. España: Desclée de Brower.

Martínez-González, J. M., Vilar-López, R., Becońa, E., y Verdejo-García, A. (2016). Self-Deception as a Mechanism for the Maintenance of Drug Addiction, Psicothema, 28, 13-19. doi: http://doi: 10.7334/psicothema2015.139.

Martínez-González, J. M., Vilar-López, R., y Verdejo-García, A. (2018). Efectividad a largo plazo de la Terapia de Grupo Cognitivo-Conductual del Alcoholismo: impacto de la patología dual en el resultado del tratamiento. Clínica y Salud, 29, 1-8. http://dx.doi.org/10.5093/clysa2018a1

Marset, M. y Ritter, C. (2004). Características de la personalidad del drogodependiente: implicaciones para nuestra relación profesional. Un modelo de intervención en medio penitenciario. Revista Española de Sanidad Penitenciaria, 6, 86-89.

Organización Mundial de la Salud (2019). Clasificación estadistica internacional de enfermedades y problemas relacionados con la salud (11. ${ }^{\text {e }}$ ed., CIE-11). Recuperado de https://icd.who.int/browse11/l-m/es

Peláez, J. C., Reyes-Molón, L. y Teijeira-Levet, C. (2013). ¿Necesitamos el tratamiento farmacológico en el trastorno de la personalidad? Acción psicológica, 10, 97-108. doi: http://dx.doi.org/10.5944/ap.10.1.7037.

Perry, J. y Bond, M. (2002). Estudios empíricos sobre la psicoterapia en los trastornos de la personalidad. En J.G. Gunderson y Gabbard (comps.), Psicoterapia en los trastornos de la personalidad (pp. 1-31). Barcelona: Ars Médica.

Reich, J. H. y Vasile, R. G. (1993). Effect of Personality Disorders on the Treatment Outcome of Axis 1 Conditions: An Update. The Journal of Nervous and Mental Disease, 181, 475-484.

Rounsaville, B.J., Dolinsky, Z.S., Sabor, T.F. y Meyer, R.E. (1987). Psychopathology as Predictor of Treatment Outcome in Alcoholics. Archives of General Psychiatry, $44,505-513$. 
Simon, W. y Lambert, M. J. (2010). Los efectos de las psicoterapias en los pacientes con trastornos de la personalidad. En A. Belloch y H. Fernández-Álvarez (eds.), Tratado de los trastornos de la personalidad (pp. 389-400) Espańa: Síntesis.

Verheul, R., Bosch, L. van den, y Ball, S. (2007). Abuso de sustancias. En J. M. Oldham, A. E. Skodol y D. Bender (dir.), Tratado de los trastornos de la personalidad (pp. 465-478). Barcelona: Elseiver Masson. 